\title{
A randomised four-intervention crossover study investigating the effect of carbohydrates on daytime profiles of insulin, glucose, non-esterified fatty acids and triacylglycerols in middle-aged men
}

\author{
Audrey E. Brynes ${ }^{1}$, C. Mark Edwards ${ }^{2}$, Mohammed A. Ghatei ${ }^{2}$, Anne Dornhorst ${ }^{2}$, Linda M. Morgan ${ }^{3}$, \\ Stephen R. Bloom ${ }^{2}$ and Gary S. Frost ${ }^{1} *$ \\ ${ }^{1}$ Nutrition and Dietetic Research Group, Hammersmith Hospital, Du Cane Road, London W12 OHS, UK \\ ${ }^{2}$ Endocrine Unit, Imperial College School of Medicine, Hammersmith Hospital, Du Cane Road, London W12 OHS, UK \\ ${ }^{3}$ School of Biomedical and Life Sciences, University of Surrey, Guildford GU2 7XH, UK
}

(Received 22 March 2002 - Revised 17 September 2002 - Accepted 22 September 2002)

\begin{abstract}
Postprandial concentrations of glucose, insulin and triacylglycerols (TG) correlate to risk for CHD. Carbohydrates affect many metabolites that could have a potential effect on cardiovascular risk factors. The objective of the present study was to examine, using a randomised prospective study, the acute (day 1) and ad libitum medium-term (day 24) effects of four diets: a high-fat diet (HIGH-FAT; $50 \%$ fat, $>34 \%$ monounsaturated fatty acids); a low-glycaemic index (GI) diet (LOW-GI; high-carbohydrate, low-GI); a high-sucrose diet (SUCROSE; high carbohydrate increase of $90 \mathrm{~g}$ sucrose/d); a high-GI diet (HIGH-GI; high-carbohydrate, high-GI). Daytime profiles ( $8 \mathrm{~h}$ ) (breakfast, lunch and tea) of lipid and carbohydrate metabolism were completed during day 1 and day 24. Seventeen middle-aged men with one or more cardiac risk factors completed the study. There was no change from day 1 or between diets in fasting glucose, lipids or homeostatic assessment model (HOMA) on day 24. The HIGH-FAT compared with the three high-carbohydrate diets was associated with lower postprandial insulin and glucose but higher postprandial TG and non-esterified fatty acids (NEFA). There was a significant increase in the $6 \mathrm{~h}$ (15.00 hours) TG concentration (day 1, 2.6 (SEM 0.3) $\mathrm{mmol} / \mathrm{l} v$. day 24, $3 \cdot 3$ (SEM 0.3) mmol/l; $P<0 \cdot 01$ ) on the SUCROSE diet. Postprandial HOMA (i.e. incremental area under the curve (IAUC) glucose $(\mathrm{mmol} / \mathrm{l}$ per $\mathrm{min}) \times$ IAUC insulin $/ 22.5(\mathrm{mU} / \mathrm{l}$ per min $)$ ) median changes from day 1 to day 24 were $-61,-43,-20$ and $+31 \%$ for the HIGH-FAT, LOW-GI, SUCROSE and HIGH-GI diets respectively. The HIGH-GI percentage change was significantly different from the other three diets $(P<0 \cdot 001)$. Despite being advised to maintain an identical energy intake there was a significant weight change $(-0.27$ (SEM 0.3$) \mathrm{kg} ; P<0.02$ ) on the LOW-GI diet compared with the SUCROSE diet (+0.84 (SEM 0.3) kg). In conclusion the HIGH-FAT diet had a beneficial effect on postprandial glucose and insulin over time but it was associated with higher postprandial concentrations of TG and NEFA. Conversely the HIGH-GI diet appeared to increase postprandial insulin resistance over the study period.
\end{abstract}

Insulin resistance: Postprandial homeostatic assessment model: High-sucrose diet:

High-fat diet: Glycaemic index

CHD remains the major cause of death in the Western world (Murray \& Lopez, 1997), despite modest falls in CHD mortality over the last three decades (Gillum, 1994). The insulin resistance syndrome is increasingly recognised as an important risk factor for CHD (Reaven,
1995). Several epidemiological studies have demonstrated an association between hyperinsulinaemia, a surrogate maker of insulin resistance, obesity, and CHD risk (Fontbonne et al. 1991; McKeigue et al. 1993; Despres et al. 1996).

\footnotetext{
Abbreviations: GI, glycaemic index; HIGH-FAT, high-fat intervention; HIGH-GI, high-carbohydrate high-glycaemic index intervention; HOMA, homeostatic assessment model; HOMA-PP, postprandial homeostatic assessment model; IAUC, incremental area under the curve; LOW-GI, highcarbohydrate low-glycaemic index intervention; MUFA, monounsaturated fatty acids; NEFA, non-esterified fatty acids; SUCROSE, high-sucrose intervention; TG, triacylglycerol.

* Corresponding author: Dr Gary Frost, fax +44 208383 3379, email g.frost@ic.ac.uk
} 
Current UK dietary advice given for cardiac protection emphasises low-fat, high-carbohydrate foods with five or more portions of fruit and vegetables a day without specifying the type of carbohydrate to use when replacing dietary fat (Department of Health, 1994).

Low-fat diets are by default high-carbohydrate diets; however concern exists about increasing carbohydrate intake above $55 \%$ as deleterious effects have been found on serum triacylglycerols (TG) and HDL-cholesterol (Parks \& Hellerstein, 2001). The inclusion of sucrose in the increasingly large quantities that are now being consumed in the Western world (moderate intake around $75 \mathrm{~g} / \mathrm{d}$; top end of the range as high as $200 \mathrm{~g} / \mathrm{d}$ ) further compounds this concern (for a review, see Frayn \& Kingman, 1995).

Diets with a high olive oil (monounsaturated fat) content have been found to produce a CHD protection effect. Cardiac risk factors have been reported to be improved on high-monounsaturated-fat diets compared with diets in which more than $55 \%$ of their energy is derived from carbohydrate (Garg et al. 1994; Reaven, 1997).

There are a wide variety of dietary carbohydrates and different classifications have been used to describe them. The 'glycaemic index' (GI) is a useful way of predicting their physiological effects. High-GI foods give higher postprandial blood glucose concentrations than those with a low GI (Jenkins et al. 1981; Foster-Powell \& Miller, 1995). Application of the GI has proved beneficial in the dietary management of diabetes, and a recent review of clinical studies has shown that low-GI carbohydrates improve glycaemic control and lipid profiles in type 2 diabetes, as well as in those at risk of CHD (Ludwig, 2002). The Food and Agriculture Organization/World Health Organization Expert Consultation report (1998) now endorses the use of the GI for categorising dietary carbohydrates in recognition that it provides useful metabolic information on carbohydrate.

Although there is strong epidemiological evidence to suggest that low-GI diets are cardio-protective (Frost et al. 1999; Hu et al. 2000; Liu et al. 2000), it is unknown whether they have any effect before the onset of CHD, or whether such diets could slow the onset of CHD.

The present prospective, randomised crossover study was designed to investigate the acute and medium-term effects of three different high-carbohydrate diets: a highcarbohydrate high-GI diet (HIGH-GI), a high-sucrose diet (SUCROSE) and a high-carbohydrate low-GI diet (LOWGI) compared with a low-carbohydrate high-fat $(>35 \%$ monounsaturated) diet (HIGH-FAT) in healthy middleaged men, with risk factors for CHD but before the progression of disease, on day profiles of insulin, glucose and lipids.

\section{Research design and methods}

The study population targeted were moderately overweight middle-aged men who were currently healthy but had one or more cardiac risk factors: BMI $>25 \mathrm{~kg} / \mathrm{m}^{2}$; raised total cholesterol:HDL $>5$; increased visceral adiposity (waist $>1000 \mathrm{~mm}$ ). Twenty-five men were approached to take part in the study. After initial screening twenty-two men were recruited. Seventeen men completed all four dietary interventions (three left the area and two were smokers who could not complete the day profile without smoking). No subjects were taking any medication or diet supplements. All had a normal electrocardiogram and physical examination. The mean age was 45 (SD 8) years, BMI 29.3 (SD 4.0) $\mathrm{kg} / \mathrm{m}^{2}$, waist:hip 0.95 (SD 0.06), waist 1050 (SD 126) $\mathrm{mm}$. Eight were non-smokers five ex-smokers and four current smokers. All reported stable body weights for the previous 3 months. The group had an 11.4 (SD 9.2) \% 10-year risk of developing CHD, as calculated by the Framingham CHD risk assessment programme (Wood et al. 1998).

Alcohol intake and physical activity level or baseline dietary intake was not different between subjects and alcohol intake and physical activity level (as assessed by previous $7 \mathrm{~d}$ recall) did not differ over the study period. Imperial College's School of Medicine, Hammersmith Campus research and ethics committee granted ethical approval. All volunteers gave written consent.

\section{Experimental protocol}

A randomised Williams Latin Square crossover study design was undertaken. During a week run-in period subjects recorded their normal habitual diet for $7 \mathrm{~d}$, after which participants were randomised to one of four $24 \mathrm{~d}$ treatments: HIGH-FAT, LOW-GI, SUCROSE or HIGHGI. The duration on each intervention was 24 (SEM 1) d. There was a minimum 3-week washout period between the diets (24 (SEM 2) d). Therefore, overall, each subject was enrolled on the study for about 7 months.

\section{Day profiles}

All studies were performed in the Clinical Investigation Unit, Hammersmith Hospital from around 8.00 hours after an overnight fast. An intravenous cannula was inserted into the antecubetal fossa for blood sampling. A breakfast meal was given at 0 min ( 9.00 hours), lunch at $180 \mathrm{~min}$ (12.00 hours) and afternoon tea at $360 \mathrm{~min}$ (15.00 hours). Blood samples were taken at $-15,0,15$, $30,45,60,120,180,195,210,225,240,300,360,375$, $390,405,420$ and $480 \mathrm{~min}$. The meals were consumed within $10 \mathrm{~min}$ on each occasion. All of the profile meals were identical in energy. Every profile meal within each intervention was matched for energy and macronutrients (Table 1a and 1b). This allowed the assessment of the cumulative meal effect of each diet. Each dietary period included a day profile on day 1 and day 24. We used identical day profiles (day 1 and 24) in order to assess the adaptation effect of ingesting a diet rich in fat $v$. sucrose $v$. high-GI $v$. low-GI choices. GI values were calculated as previously described (Frost et al. 1994) using bread as the standard. Values given in parentheses in Tables 1 and 2 are calculated with glucose as the standard (FosterPowell \& Miller, 1995). 
Table 1a. Composition of breakfast, lunch and afternoon tea meals of identical energy and differing only in the glycaemic index (Gl) of the carbohydrate were consumed on day 1 and 24 of the corresponding intervention by healthy middle-aged men ( $n 17)$

\begin{tabular}{|c|c|c|}
\hline & HIGH-GI day profile & LOW-GI day profile \\
\hline \multirow[t]{6}{*}{ Breakfast } & Weetabix (40 g) & All-bran $(60 \mathrm{~g})$ \\
\hline & Semi-skimmed milk (250 ml) & Semi-skimmed milk $(250 \mathrm{ml})$ \\
\hline & Decaffeinated coffee (200 ml) & Decaffeinated coffee (200 ml) \\
\hline & Wholemeal bread $(60 \mathrm{~g})$ & Rye bread $(60 \mathrm{~g})$ \\
\hline & $70 \%$ fat spread $(18 \mathrm{~g})$ & $70 \%$ fat spread $(18 \mathrm{~g})$ \\
\hline & Glucose (15 g) & Jam (16 g) \\
\hline Energy (kJ) & 2310 (550 kcal) & 2310 (550 kcal) \\
\hline Protein (\%E) & 13 & 16 \\
\hline Carbohydrate (\%E) & 54 & 52 \\
\hline GI & $102\left(71^{\star}\right)$ & $68\left(48^{\star}\right)$ \\
\hline Total fat (\%E) & 31 & 32 \\
\hline MUFA:PÜFA:SFA & $8: 10: 8$ & $8: 10: 8$ \\
\hline \multirow[t]{5}{*}{ Lunch } & Chicken curry (175 g) & Chicken curry $(150 \mathrm{~g})$ \\
\hline & Instant potato $(250 \mathrm{~g})$ & Parboiled rice $(200 \mathrm{~g})$ \\
\hline & Glucose $(25 \mathrm{~g})$ & \\
\hline & Fresh orange juice $(80 \mathrm{ml})$ & Peeled satsuma (130 g) \\
\hline & Water $(120 \mathrm{ml})$ & Water (200 ml) \\
\hline Energy (kJ) & 2310 (550 kcal) & 2310 (550 kcal) \\
\hline Protein (\%E) & 17 & 16 \\
\hline Carbohydrate (\%E) & 50 & 51 \\
\hline GI & $118\left(83^{*}\right)$ & $68\left(48^{\star}\right)$ \\
\hline Total fat (\%E) & 34 & 33 \\
\hline MUFA:PÜFA:SFA & $16: 7: 12$ & $13: 5: 10$ \\
\hline \multirow[t]{7}{*}{ Afternoon tea } & Müller light yoghurt $(200 \mathrm{~g})$ & Müller light yoghurt (200 g) \\
\hline & Semi-skimmed milk (100 ml) & Semi-skimmed milk (100 ml) \\
\hline & Decaffeinated coffee $(200 \mathrm{ml})$ & Decaffeinated coffee $(200 \mathrm{ml})$ \\
\hline & Glucose powder (25 g) & $\operatorname{Jam}(40 \mathrm{~g})$ \\
\hline & Low-fat cheese $(17 \mathrm{~g})$ & Low-fat cheese $(17 \mathrm{~g})$ \\
\hline & Low-fat spread $(9 \mathrm{~g})$ & Low-fat spread $(9 \mathrm{~g})$ \\
\hline & Low-fat digestives (three) & Oatcakes (four) \\
\hline Energy (kJ) & 2310 (550 kcal) & 2310 (550 kcal) \\
\hline Protein (\%E) & 13 & 14 \\
\hline Carbohydrate (\%E) & 53 & 51 \\
\hline GI & $88\left(62^{\star}\right)$ & $69\left(48^{\star}\right)$ \\
\hline Total fat (\%E) & 34 & 35 \\
\hline MUFA:PÜA:SFA & $11: 3: 19$ & $6: 1: 14$ \\
\hline
\end{tabular}

HIGH-GI, high-carbohydrate high-GI diet, LOW-GI, high-carbohydrate low-GI diet; \%E, percentage of energy; MUFA, monounsaturated fatty acids, PUFA, polyunsaturated fatty acids, SFA, saturated fatty acids.

${ }^{*} \mathrm{Gl}$ calculated with glucose as the standard.

\section{Medium-term dietary interventions}

Based on the results from previous studies, alterations in glycaemia, insulinaemia and lipidaemia were assumed to occur within the first 3 weeks after crossover (Coulston et al. 1987, 1989; Mensink \& Katan, 1990). The dietary aim of the medium-term intervention was to include a food from the current diet with each meal while keeping the energy intake constant. Written dietary advice was provided, along with $500 \mathrm{ml}$ olive oil (for HIGH-FAT), $2 \mathrm{~kg}$ sugar (for SUCROSE), $700 \mathrm{~g}$ instant potato (for HIGHGI) or $1200 \mathrm{~g}$ wholegrain rye bread (for LOW-GI) to aid compliance over the $24 \mathrm{~d}$ period. The meals provided on the day 1 profile were used to reinforce the advice given. Similar exercise and daily routines were encouraged throughout the study. Subjects were requested to have no alcohol and take no exercise on the day before the day profiles. A $7 \mathrm{~d}$ food diary using household measures on the last $7 \mathrm{~d}$ of the intervention monitored compliance. Monitoring was also undertaken by telephone contact during each intervention.

\section{Outcome measures}

Weight, fasting lipids, postprandial insulin, glucose, and lipid responses were measured. A computerised version of homeostatic assessment model (HOMA) was used to measure change in fasting insulin sensitivity using fasting insulin (pmol/l) and glucose concentrations (mmol/l) (Turner et al. 1979; Levy et al. 1998). An index of postprandial insulin resistance (HOMA-PP) was obtained by using the following equation: HOMA-PP $\left(10^{3}\right)=$ incremental area under the curve (IAUC) glucose $(\mathrm{mmol} /$ 1 per $\mathrm{min}) \times$ IAUC insulin $/ 22.5(\mathrm{mU} / \mathrm{l}$ per $\mathrm{min})$ where insulin is reported in international units $(\mathrm{pmol} / 7 \cdot 5=\mathrm{mU} / \mathrm{l})$ (Raben et al. 2001).

\section{Laboratory analysis}

Blood samples were collected into lithium heparin tubes for the measurement of TG, non-esterified fatty acids (NEFA) and glucose or lithium heparin tubes containing 4000 kIU aprotinin ('Trasylol'; Bayer, Newbury, Berks, 
Table 1b. Composition of breakfast, lunch and afternoon tea meals of identical energy and differing only in the fat and sucrose content were consumed on day 1 and 24 of the corresponding intervention by healthy middle-aged men $(n 17)$

\begin{tabular}{|c|c|c|}
\hline & HIGH-FAT day profile & High-SUCROSE day profile \\
\hline \multirow[t]{6}{*}{ Breakfast } & Weetabix $(20 \mathrm{~g})$ & Weetabix $(40 \mathrm{~g})$ \\
\hline & $\begin{array}{l}\text { Full-fat milk }(230 \mathrm{ml}) \\
\text { Double cream }(20 \mathrm{ml})\end{array}$ & Semi-skimmed milk (250 ml) \\
\hline & Decaffeinated coffee $(200 \mathrm{ml})$ & Decaffeinated coffee $(200 \mathrm{ml})$ \\
\hline & Wholemeal bread $(30 \mathrm{~g})$ & Wholemeal bread $(30 \mathrm{~g})$ \\
\hline & $70 \%$ fat spread $(27 \mathrm{~g})$ & $70 \%$ fat spread $(18 \mathrm{~g})$ \\
\hline & Low-sugar jam $(8 \mathrm{~g})$ & Sugar $(30 \mathrm{~g})$ \\
\hline Energy $(\mathrm{kJ})$ & $2310(550 \mathrm{kcal})$ & 2310 (550 kcal) \\
\hline Protein (\%E) & 9 & 11 \\
\hline Carbohydrate (\%E) & 28 & 57 \\
\hline GI & $86\left(60^{\star}\right)$ & $92\left(64^{*}\right)$ \\
\hline Total fat (\%E) & 63 & 30 \\
\hline MUFA:PÜFA:SFA & $18: 15: 24$ & $8: 10: 8$ \\
\hline \multirow{5}{*}{ Lunch } & Chicken curry $(175 \mathrm{~g})$ & Chicken curry $(175 \mathrm{~g})$ \\
\hline & Parboiled rice $(120 \mathrm{~g})$ & Parboiled rice $(120 \mathrm{~g})$ \\
\hline & Olive oil $(10 \mathrm{~g})$ & Sugar $(30 \mathrm{~g})$ \\
\hline & Sugar-free squash $(10 \mathrm{ml})$ & Sugar-free squash $(10 \mathrm{ml})$ \\
\hline & Water (200 ml) & Water (200 ml) \\
\hline Energy $(\mathrm{kJ})$ & 2310 (550 kcal) & 2310 (550 kcal) \\
\hline Protein (\%E) & 17 & 16 \\
\hline Carbohydrate (\%E) & 30 & 50 \\
\hline GI & $70\left(49^{*}\right)$ & $79\left(55^{\star}\right)$ \\
\hline Total fat (\%E) & 54 & 35 \\
\hline MUFA:PÜFA:SFA & $21: 15: 15$ & 13:6:12 \\
\hline \multirow[t]{7}{*}{ Afternoon tea } & Müller light yoghurt ( $100 \mathrm{~g})$ & Müller light yoghurt $(200 \mathrm{~g})$ \\
\hline & Full-fat milk $(100 \mathrm{ml})$ & Semi-skimmed milk (100 ml) \\
\hline & Decaffeinated coffee $(200 \mathrm{ml})$ & Decaffeinated coffee (200 ml) \\
\hline & Double cream $(25 \mathrm{ml})$ & Sugar (25g) \\
\hline & Full-fat cheese $(17 \mathrm{~g})$ & Low-fat cheese $(17 \mathrm{~g})$ \\
\hline & $70 \%$ fat spread $(9 \mathrm{~g})$ & $40 \%$ fat spread $(9 \mathrm{~g})$ \\
\hline & Digestives (three) & Low-fat digestives (three) \\
\hline Energy $(\mathrm{kJ})$ & 2310 (550 kcal) & 2310 (550 kcal) \\
\hline Protein (\%E) & 11 & 13 \\
\hline Carbohydrate (\%E) & 30 & 53 \\
\hline $\mathrm{Gl}$ & $71\left(50^{\star}\right)$ & $75\left(53^{*}\right)$ \\
\hline Total fat (\%E) & 60 & 34 \\
\hline MUFA:PÜFA:SFA & 21:9:34 & 11:3:19 \\
\hline
\end{tabular}

HIGH-FAT, $50 \%$ fat (>34\% monounsaturated fat) diet; SUCROSE, high-sucrose diet with high carbohydrate increase of $90 \mathrm{~g}$ sucrose/d; \%E, percentage of energy; GI, glycaemic index; MUFA, monounsaturated fatty acids; PUFA, polyunsaturated fatty acids; SFA, saturated fatty acids.

${ }^{*} \mathrm{Gl}$ calculated with glucose as the standard.

UK) for the measurement of insulin. Samples were centrifuged immediately at $3200 \mathrm{rpm}$ for $10 \mathrm{~min}$. The plasma was then portioned into plain plastic $2.5 \mathrm{ml}$ tubes. These were immediately frozen and stored at $-20^{\circ} \mathrm{C}$ until analysis. Plasma glucose concentrations were measured using a glucose-oxidase-based autoanalyser (Technicon, Axon Bayer Diagnostic, Newbury, Berkshire, UK). Insulin concentrations were measured in-house using a specific radioimmunoassay (Albano et al. 1972). The inter-assay and intra-assay $\mathrm{CV}$ were less than $10 \%$. The assay was capable of detecting $2 \mathrm{pmol} / \mathrm{l}$ with $95 \%$ confidence. All samples were included in one assay and analysed in duplicate on the first freeze-thaw. TG and NEFA were measured by the enzymic colorimetric method using commercial kits (MPR2 Triacylglycerols GPO-PAP 701912 kit; Boehringer Mannheim, Newbury, Berkshire, UK; NEFA kit; WAKO Chemicals USA, Inc., Alpha Eastleigh, Hampshire, $\mathrm{UK}), \mathrm{CV}<6 \%$, and $<3 \%$ respectively. Cholesterol and HDL-cholesterol were measured on a DAX-72 Analyser (Bayer Diagnostics, Basingstoke, Hampshire, UK).

\section{Statistical analysis}

Sample size was estimated from previous studies using CHD patients and a power of $80 \%$ and alpha $5 \%$ with an estimated difference for insulin between IAUC of $1000 \mathrm{pmol} \times \mathrm{min} / \mathrm{l}$ and a standard deviation of $1000 \mathrm{pmol} \times \mathrm{min} / 1$. This suggested a sample size of twenty pairs. All results are presented as the mean and standard error of the mean unless stated otherwise. In Table 2 a repeated-measures design, with one repeatedmeasures factor: diet (HIGH-FAT, HIGH-GI, SUCROSE, LOW-GI) was used. For the rest of the tables a repeatedmeasures design with a $2 \times 2$ factorial structure for the repeated measures, with factors diet (HIGH-FAT, HIGHGI, SUCROSE, LOW-GI) and day (1 and 24) was used. Individual $P$ values have only been reported if there was a significant interaction using the post hoc TukeyKramer test. A log transformation was used for positive skewed variables. The Friedman test was used for variables that did not $\log$ transform. $P<0.05$ was taken as significant. 
Table 2. Compliance to dietary intervention monitored by $7 \mathrm{~d}$ food diaries in middle-aged men $(n 17) \S$ (Mean values and standard errors of the mean)

\begin{tabular}{|c|c|c|c|c|c|c|c|c|}
\hline \multirow[b]{2}{*}{ Intervention... } & \multicolumn{2}{|c|}{ HIGH-FAT } & \multicolumn{2}{|c|}{ LOW-GI } & \multicolumn{2}{|c|}{ SUCROSE } & \multicolumn{2}{|c|}{ HIGH-GI } \\
\hline & Mean & SEM & Mean & SEM & Mean & SEM & Mean & SEM \\
\hline Weight change (kg) & +0.46 & 0.3 & $-0.27 \dagger$ & 0.3 & +0.84 & 0.3 & +0.43 & 0.3 \\
\hline Energy (MJ) & $10 \cdot 9 \ddagger$ & 0.51 & 7.82 & 0.45 & 9.9 & 0.51 & 9.02 & 0.34 \\
\hline Fat (\%E)\| & $47 \cdot 0^{\star}$ & $1 \cdot 2$ & $33 \cdot 4$ & $1 \cdot 1$ & 33.2 & $1 \cdot 0$ & 35.9 & $1 \cdot 2$ \\
\hline Monounsaturated fatty acids $\|$ & $16 \cdot 1^{\star}$ & 0.7 & $9 \cdot 0$ & 0.5 & $9 \cdot 0$ & 0.5 & $10 \cdot 0$ & $0 . \overline{6}$ \\
\hline Carbohydrate (\%E) & $35 \cdot 9^{\star}$ & 1.4 & $47 \cdot 1$ & 1.4 & $51 \cdot 0$ & 1.6 & $46 \cdot 4$ & 1.5 \\
\hline Starch\| & $21 \cdot 1$ & $1 \cdot 1$ & $25 \cdot 1$ & $1 \cdot 2$ & $21 \cdot 8$ & $1 \cdot 3$ & $23 \cdot 2$ & $1 \cdot 7$ \\
\hline Fibre $(g) \emptyset$ & 22 & 2 & $31^{*}$ & 4 & 19 & 2 & 19 & 1 \\
\hline Sucrose $(g) \|$ & 51 & 5 & 45 & 5 & $132^{*}$ & 12 & 46 & 7 \\
\hline GI\| & 87 & 1.4 & $69^{*}$ & $1 \cdot 3$ & 88 & 1.4 & $97^{*}$ & $1 \cdot 3$ \\
\hline GI (glucose as standard)\| & 61 & & $48^{\star}$ & & 62 & & $68^{*}$ & \\
\hline
\end{tabular}

HIGH-FAT, $50 \%$ fat (>34\% monounsaturated fat) diet; LOW-GI, high-carbohydrate low glycaemic index diet; HIGH-GI, high carbo-

hydrate high-GI diet; SUCROSE, high sucrose diet with high carbohydrate increase of $90 \mathrm{~g}$ sucrose/d; \%E, percentage of energy.

* Mean value was significantly different from those for the other three interventions $(P<0.05)$.

† Mean value for LOW-GI was significantly different from that for SUCROSE $(P<0.02)$.

$\ddagger$ Mean value for HIGH-FAT was significantly different from those for LOW-GI and HIGH-GI $(P<0.05)$.

$\S$ For details of diets and procedures, see Table 1 and p. 208.

|| Data log-transformed to perform statistical analysis.

I Southgate method.

IAUC above baseline was calculated using the trapezoidal rule.

\section{Results}

\section{Ad libitum compliance}

All subjects reported $100 \%$ compliance by returning the empty containers or leftover key foods. Subjects returned five $7 \mathrm{~d}$ diet diaries. These were analysed using household portion measures, and suggested that each dietary intervention was achieved (Table 2). No one was excluded due to poor reporting. There was a significant difference between the endpoints of the LOW-GI and HIGH-GI interventions. The HIGH-GI diet increased GI from a baseline value of 87 (SEM 2) to 97 (SEM 1) $(P<0 \cdot 001)$ and on the LOWGI diet the GI fell to 69 (SEM 1) $(P<0.001)$. There was a mean increase of $90 \mathrm{~g}$ sucrose/d on the high-SUCROSE diet and the HIGH-FAT intervention raised the baseline intake of fat from 36.2 (SEM 1.3) to 47.0 (SEM 1.2) \% energy, over half of which came from monounsaturated fatty acids (MUFA). There was a significant increase in dietary fibre on the LOW-GI diet (Table 2).

\section{Weight}

Despite being advised to maintain an identical energy intake and steady weight over the study period there was a spontaneous increase in energy intake on the HIGHFAT intervention relative to the three high-carbohydrate interventions. There was a trend for the lowest energy intake to be consumed on the LOW-GI diet and this resulted in a small but significant weight loss compared with the high-SUCROSE diet (Table 2) ( -0.27 (SEM 0.3) v. 0.84 (SEM 0.3$) \mathrm{kg} ; P<0.02)$.

\section{Fasting measurements}

Fasting HOMA demonstrated no change in insulin sensitivity on any intervention over all of the four study periods. There was no change in any of the fasting parameters measured on any of the diets over time or between the diets (Table 3 ).

\section{Day 1 between and within interventions}

Glucose. No dietary intervention was associated with any significant differences in fasting glucose concentrations on day 24 (Table 3 ). The total day IAUC glucose was similar for the four interventions (Table 4). However the individual meal responses to the HIGH-FAT breakfast was significantly lower than for the three high-carbohydrate breakfasts $(P<0.02)$ (Table 4 ; Fig. 1). At lunch and tea the glucose response to the HIGH-GI intervention was higher $(P<0.05$ and $P<0.01$, respectively) than the LOW-GI. As expected the maximum glucose concentration was lowest after the HIGH-FAT $<$ LOW-GI $<$ SUCROSE $<$ HIGH-GI meals. This same pattern was seen at breakfast, lunch and tea. In all four interventions the maximum glucose concentration response was greatest following breakfast, with lunch and tea (which were matched for energy and macronutrients) showing an attenuated response $(P<0.002)$ (Table 5; Fig. 1). The time of the highest concentration, measured in min from start of consumption of each meal, did not differ within each intervention.

Insulin. Total IAUC insulin was significantly lower after HIGH-FAT $(P<0.001)$ (Table 4; Fig. 2). The total IAUC insulin on the LOW-GI intervention was less than HIGH-GI $(P<0.05)$ (Table 4$)$. The insulin response to HIGH-FAT was lower at breakfast and lunch but not tea. Insulin response to the HIGH-GI intervention at lunch was significantly higher than LOW-GI, as it was for tea and the insulin IAUC was also significantly higher than SUCROSE or HIGH-FAT at tea. Similar to the effect on glucose the maximum insulin concentration was lowest after the HIGH-FAT $<$ LOW-GI $<$ SUCROSE $<$ 
Table 3. Fasting lipid and homeostatic assessment model (HOMA) results of seventeen middleaged men on day $24^{*} \dagger$

(Mean values and standard errors of the mean)

\begin{tabular}{|c|c|c|c|c|c|c|c|c|}
\hline \multirow[b]{2}{*}{ Intervention. .. } & \multicolumn{2}{|c|}{ HIGH-FAT } & \multicolumn{2}{|c|}{ LOW-GI } & \multicolumn{2}{|c|}{ SUCROSE } & \multicolumn{2}{|c|}{ HIGH-GI } \\
\hline & Mean & SEM & Mean & SEM & Mean & SEM & Mean & SEM \\
\hline TC (mmol/l) & $5 \cdot 2$ & 0.3 & $5 \cdot 2$ & 0.2 & $5 \cdot 3$ & 0.2 & $5 \cdot 0$ & 0.2 \\
\hline TC:HDL & 5.5 & 0.5 & $5 \cdot 8$ & 0.5 & $5 \cdot 8$ & 0.4 & 5.5 & 0.6 \\
\hline $\mathrm{HDL}(\mathrm{mmol} / \mathrm{l})$ & 1.01 & 0.1 & 0.96 & 0.1 & 0.96 & 0.1 & 0.99 & 0.1 \\
\hline LDL (mmol/l) & 3.4 & 0.2 & 3.5 & 0.3 & 3.5 & 0.2 & 3.3 & 0.2 \\
\hline $\mathrm{TG}(\mathrm{mmol} / \mathrm{l})$ & 1.5 & 0.2 & $1 \cdot 7$ & 0.2 & $1 \cdot 7$ & 0.2 & 1.7 & 0.2 \\
\hline NEFA $(\mathrm{mmol} / \mathrm{l})$ & 0.45 & 0.1 & 0.65 & 0.1 & 0.52 & 0.1 & 0.6 & 0.1 \\
\hline Glucose $(\mathrm{mmol} / \mathrm{l})$ & 5.4 & 0.2 & $5 \cdot 4$ & 0.1 & 5.4 & 0.1 & $5 \cdot 2$ & 0.1 \\
\hline Insulin (pmol/l) & 71 & 11 & 85 & 19 & 90 & 21 & 80 & 9 \\
\hline HOMA (\%S) & 99 & 13 & 114 & 24 & 111 & 24 & 91 & 17 \\
\hline
\end{tabular}

HIGH-FAT, $50 \%$ fat (>34\% monounsaturated fat) diet; LOW-GI, high-carbohydrate low glycaemic index diet; HIGH-GI, high-carbohydrate high-glycaemic index diet; SUCROSE, high-sucrose diet with high carbohydrate increase of $90 \mathrm{~g}$ sucrose/d; TC, total cholesterol; TG, triacylglycerol; NEFA, non-esterified fatty acids; \%S, percentage change in insulin sensitivity.

${ }^{*}$ For details of diets and procedures, see Table 1 and p. 208.

†There were no differences between the four diets at baseline (data not shown) or after $24 \mathrm{~d}$ on each intervention diet (change from baseline ANOVA).

Table 4. Postprandial results on day 1 (acute) and change between day 1 and day 24 in seventeen middle-aged men after consuming three meals that were rich in fat, low-glycaemic, high in sucrose or high-glycaemic, all containing $2310 \mathrm{~kJ}$ and matched for macronutrient content||

(Mean values and standard errors of the mean)

\begin{tabular}{|c|c|c|c|c|c|c|c|c|c|}
\hline \multirow[b]{2}{*}{ Intervention... } & \multicolumn{2}{|c|}{ HIGH-FAT } & \multicolumn{2}{|c|}{ LOW-GI } & \multicolumn{2}{|c|}{ SUCROSE } & \multicolumn{2}{|c|}{$\mathrm{HIGH}-\mathrm{Gl}$} & \multirow[b]{2}{*}{ ANOVA $(P)$} \\
\hline & Mean & SEM & Mean & SEM & Mean & SEM & Mean & SEM & \\
\hline \multicolumn{10}{|c|}{ Total IAUC $(0-480 \mathrm{~min} ; \mathrm{mmol} / \mathrm{I} \times \mathrm{min})$} \\
\hline Glucose & 352 & 65 & 432 & 77 & 442 & 99 & 493 & 70 & NS \\
\hline Insulin & $40^{*}$ & $7 \cdot 6$ & $71 \cdot 3$ & $9 \cdot 0$ & $82 \cdot 7$ & 13 & $92.5 \dagger$ & 14 & 0.0001 \\
\hline NEFA & $-44.9^{\star}$ & 28 & -176 & 29 & -159 & 28 & -200 & 42 & 0.0007 \\
\hline TG & $501^{*}$ & 99 & 302 & 61 & 331 & 50 & 185 & 90 & 0.008 \\
\hline \multicolumn{10}{|c|}{ Breakfast $(0-120 \mathrm{~min} ; \mathrm{mmol} / / \times \mathrm{min})$} \\
\hline Glucose & $150^{*}$ & 28 & 178 & 19 & 215 & 32 & 210 & 23 & 0.02 \\
\hline Insulin & $17 \cdot 5^{\star}$ & 3.2 & $26 \cdot 2$ & 3.7 & $35 \cdot 8$ & $5 \cdot 8$ & 38.8 & $7 \cdot 0$ & 0.03 \\
\hline NEFA & $-26 \cdot 7$ & $5 \cdot \overline{9}$ & -37.4 & $6 \cdot 1$ & $-35 \cdot 8$ & $6 \cdot 6$ & -37.4 & $9 \cdot 3$ & NS \\
\hline TG & $27 \cdot 2 \S$ & 6.9 & 5.9 & $5 \cdot 0$ & $23 \cdot 3$ & $7 \cdot 4$ & $6 \cdot 6$ & $8 \cdot 9$ & 0.04 \\
\hline \multicolumn{10}{|c|}{ Lunch (180-300 min; mmol/l × min) } \\
\hline Glucose & 49 & 14 & 88 & 19 & 79 & 12 & $133^{*}$ & 32 & 0.03 \\
\hline Insulin & $3.4^{\star}$ & $1 \cdot 2$ & $10 \cdot 8$ & $2 \cdot 4$ & 11.5 & $2 \cdot 0$ & $15 \cdot 8 \dagger$ & 4.4 & 0.0001 \\
\hline NEFA & $16 \cdot 8^{*}$ & $3 \cdot 1$ & $2 \cdot 8$ & 1.6 & -2.09 & $2 \cdot 9$ & -1.63 & $2 \cdot 3$ & 0.0002 \\
\hline TG & $59.8 \ddagger$ & $11 \cdot 7$ & 48.9 & $8 \cdot 6$ & $38 \cdot 2$ & $10 \cdot 8$ & $28.8 \dagger$ & 5.9 & 0.002 \\
\hline \multicolumn{10}{|c|}{ Tea $(360-480 \mathrm{~min} ; \mathrm{mmol} / / \mathrm{l} \mathrm{min})$} \\
\hline Glucose & 98 & 13 & 100 & 27 & 101 & 18 & $166^{*}$ & 22 & 0.03 \\
\hline Insulin & $9 \cdot 4$ & $1 \cdot 7$ & $6 \cdot 2$ & 1.5 & $11 \cdot 3$ & $2 \cdot 5$ & $17 \cdot 4^{*}$ & 3.5 & 0.002 \\
\hline NEFA & $-15 \cdot 8$ & 3.7 & $-7 \cdot 0$ & $2 \cdot 7$ & $-13 \cdot 1$ & $3 \cdot 1$ & $-7 \cdot 3$ & 2.5 & NS \\
\hline $\mathrm{TG}$ & -26 & $9 \cdot 4$ & -6.5 & $10 \cdot 4$ & $-6 \cdot 1$ & $12 \cdot 3$ & 7.8 & $9 \cdot 7$ & NS \\
\hline \multicolumn{10}{|c|}{ Total IAUC change (day 1 -day $24 ; \mathrm{mmol} / \mathrm{l} \times \mathrm{min}$ ) } \\
\hline Glucose & -166 & 74 & -97 & 62 & -55 & 69 & 86 & 57 & NS \\
\hline Insulin & $10 \cdot 1$ & $6 \cdot 6$ & -1.4 & 8.6 & -18.4 & 9.5 & 14.6 & 12 & NS \\
\hline NEFA & $27 \cdot 6$ & 34 & -3.9 & 28 & $28 \cdot 3$ & 30 & 22 & 45 & NS \\
\hline TG & -20 & 98 & $29 \cdot 4$ & 62 & $76 \cdot 7$ & 35 & 143 & 87 & NS \\
\hline
\end{tabular}

HIGH-FAT, $50 \%$ fat (>34\% monounsaturated fat) diet; LOW-GI, high-carbohydrate low glycaemic index diet; HIGH-GI, high-carbohydrate high-glycaemic index diet; SUCROSE, high sucrose diet with high carbohydrate increase of $90 \mathrm{~g}$ sucrose/d; NEFA, non-esterified fatty acids; TG, triacylglycerol; IAUC, incremental area under the curve.

${ }^{*}$ Mean value was significantly different from those for the other three interventions $(P<0.05)$.

† Mean value for HIGH-GI was significantly different from that for LOW-GI $(P<0.05)$.

$\ddagger$ Mean value for HIGH-FAT was significantly different from those for SUCROSE and HIGH-GI $(P<0.01)$.

$\S$ Mean value for HIGH-FAT was significantly different from those for LOW-GI and HIGH-GI $(P<0.05)$.

\|l For details of diets and procedures, see Table 1 and p. 208. 

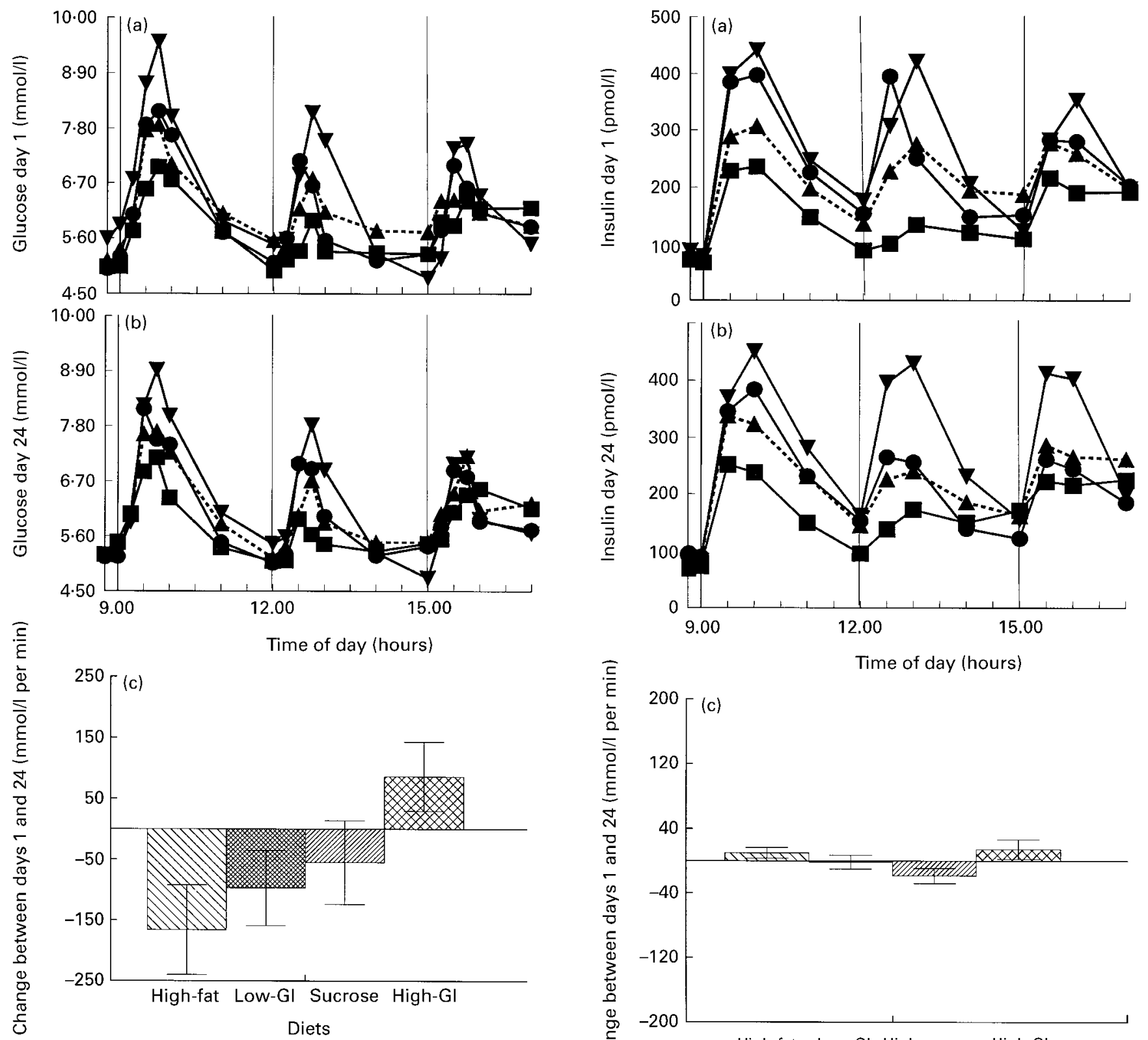

Fig. 1. Circulating plasma glucose response to three meals on day 1 (a) and day 24 (b) in men on a high-fat diet (ם), a high-glycaemic index (GI) diet $(\mathbf{\nabla})$, a high-sucrose diet $(\bullet)$ or a low-Gl diet $(\mathbf{\Lambda})$. (c), Difference between day 1 and day 24. Mean values are shown and vertical bars represent standard errors of the mean. For details of diets and procedures, see Table 1 and p. 208.

HIGH-GI meals. This same pattern was seen at breakfast, lunch and tea.

Triacylglycerols. Total IAUC TG was significantly higher on the HIGH-FAT intervention $(P<0 \cdot 01)$, and this was also the case for individual meal responses for breakfast and lunch. There were no overall total day differences between the three high-carbohydrate meals (Table 4; Fig. 3). After tea the IAUC for the HIGH-GI intervention continued to rise while the other three interventions had started to fall. The TG response to SUCROSE lay between the LOW-GI and HIGH-GI profiles.

Non-esterified fatty acids. Total IAUC NEFA was significantly different after HIGH-FAT $(P<0.001)$ compared

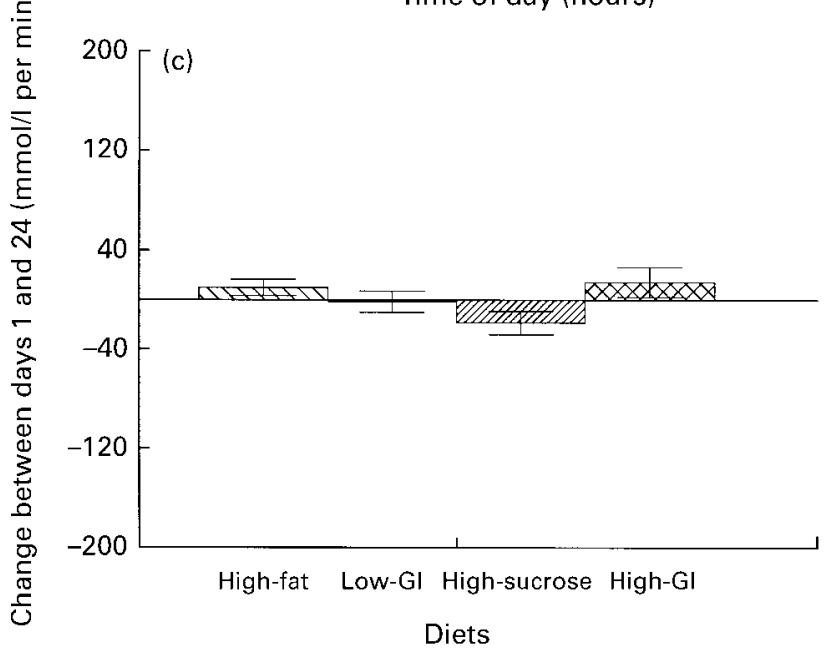

Fig. 2. Circulating plasma insulin response to three meals on day 1 (a) and day 24 (b) in men on a high-fat diet (ם), a high-glycaemic index (Gl) diet $(\mathbf{\nabla})$, a high-sucrose $\operatorname{diet}(\bullet)$ or a low-Gl diet $(\mathbf{\Lambda})$. (c), Difference between day 1 and day 24. Mean values are shown and vertical bars represent standard errors of the mean. For details of diets and procedures, see Table 1 and p. 208.

with the three carbohydrate-containing meals (Table 4; Fig. 4). Suppression of NEFA was similar after all four breakfasts, but by lunch (180-300 min) the NEFA concentration increased on the HIGH-FAT intervention while on the three high-carbohydrate interventions NEFA remained suppressed. At tea (360-480 min) there was no difference in IAUC NEFA suppression after all four interventions; however the NEFA concentration at the 360 min time point was significantly higher on the HIGH-FAT intervention (Fig. 4), therefore the area under the curve (not IAUC) was significantly higher (data not shown). 
Table 5. Maximum glucose concentration (mmol/l) on day 1 in seventeen middle-aged men after consuming three meals that were rich in fat, low glycaemic, high in sucrose or high-glycaemic§

(Mean values and standard errors of the mean)

\begin{tabular}{|c|c|c|c|c|c|c|c|c|c|}
\hline \multirow[b]{2}{*}{ Intervention... } & \multicolumn{2}{|c|}{ HIGH-FAT } & \multicolumn{2}{|c|}{ LOW-GI } & \multicolumn{2}{|c|}{ SUCROSE } & \multicolumn{2}{|c|}{ HIGH-GI } & \multirow[b]{2}{*}{ ANOVA (between diets; $P$ ) } \\
\hline & Mean & SEM & Mean & SEM & Mean & SEM & Mean & SEM & \\
\hline Breakfast & $7.5 \dagger$ & 0.4 & 8.3 & 0.4 & 8.9 & 0.4 & $9.9 \ddagger$ & 0.6 & 0.001 \\
\hline Lunch & $6 \cdot 1^{*}$ & 0.1 & $7 \cdot 0^{*}$ & 0.3 & $7 \cdot 4^{*}$ & $0.2^{*}$ & $8 \cdot 8^{*}$ & 0.4 & $<0.001$ \\
\hline Tea & $6 \cdot 8$ & 0.2 & $7 \cdot 1$ & 0.3 & 7.4 & $0 . \overline{3}$ & $8 \cdot 4^{\star}$ & 0.5 & 0.001 \\
\hline
\end{tabular}

HIGH-FAT, $50 \%$ fat (>34\% monounsaturated fat) diet; LOW-GI, high-carbohydrate low glycaemic index diet; HIGH-GI, high-carbohydrate high-glycaemic index diet; SUCROSE, high sucrose diet with high carbohydrate increase of $90 \mathrm{~g}$ sucrose/d.

* Mean value was significantly different from those for the other three interventions $(P<0.05)$.

† Mean value for HIGH-FAT was significantly different from those for SUCROSE and HIGH-GI $(P<0.01)$.

$\ddagger$ Mean value for HIGH-GI was significantly different from that of LOW-GI $(P<0.01)$.

$\S$ For details of diets and procedures, see Table 1 and p. 208.

\section{Day 24 (adaptation over time)}

On day 24, after ad libitum consumption of each diet, the differences in IAUC insulin, TG and NEFA seen on day 1 remained.

The median changes in IAUC HOMA-PP from day 1 to day 24 were $-61,-43,-20$ and $+31 \%$ (Table 6 ; Fig. 5) for the HIGH-FAT, LOW-GI, SUCROSE and HIGH-GI diets respectively. The percentage change in HOMA-PP for the HIGH-GI intervention was significantly different from the other three diets (Table $6 ; P<0 \cdot 001$ ). There was no significant difference in the relative change in total IAUC between any diet on day 24 or the percentage change between day 1 and day 24 on glucose, insulin, NEFA or TG profiles (Table 4), as measured by ANOVA.

A paired $t$ test on day $1 v$. day 24 total IAUC suggested a rise in TG after $24 \mathrm{~d}$ of SUCROSE consumption $(P<0 \cdot 01)$ and a trend towards decreased insulin total IAUC concentration after $24 \mathrm{~d}$ of SUCROSE consumption $(P=0.07)$. There was also a significant increase in the $6 \mathrm{~h}(15.00$ hours) TG concentration (day 1, 2.6 (SEM 0.3) mmol/l $v$. day $24,3 \cdot 3(\operatorname{SEM} 0 \cdot 3) \mathrm{mmol} / \mathrm{l} ; P<0 \cdot 01)$, which was not seen on any other intervention. A significant increase in TG at breakfast after $24 \mathrm{~d}$ HIGH-GI consumption $(P=0.03)$ was also noted.

\section{Discussion}

The present study addresses an important question in nutrition at the present time, namely which diet is best for the management of insulin resistance or the metabolic syndrome? We compared diets high in carbohydrate with a diet high in MUFA (HIGH-FAT). We were interested whether the source of the carbohydrate matters and thus compared three types of carbohydrate: a low-GI diet, a high-sucrose diet and a high-GI diet.

The present study investigated whether cardiovascular risk factors are modifiable by simple dietary interventions. Postprandial concentrations of glucose, insulin and TG all correlate to $\mathrm{CHD}$ risk and all can be favourably and adversely influenced by the dietary manipulation of fat and carbohydrates. After 3 weeks of each diet significant differences in postprandial metabolism and postprandial insulin resistance were seen.

Of the diets we examined, the high-glycaemic, highcarbohydrate diet was associated with the least favourable postprandial profiles, with the low-glycaemic high-carbohydrate diet having the most favourable of the three high-carbohydrate diets. By contrast, the high-MUFA diet produced favourable differences in postprandial glucose and insulin responses; however these were accompanied with adverse TG metabolism that would be predicted to negate against any other metabolic benefits. The highsucrose diet had an effect that was between that of the high- and low-glycaemic diets.

The present study in free-living volunteers achieved good dietary compliance among middle-aged subjects eating ad libitum, and making the necessary dietary changes by

Table 6. Postprandial homeostatic assessment model results for day 1 and day 24 in seventeen middle-aged men after consuming an ad libitum diet that was rich in fat, low-glycaemic index (GI), high in sucrose or high-Gl†

(Median values and $95 \%$ confidence intervals)

\begin{tabular}{|c|c|c|c|c|c|c|c|c|}
\hline \multirow[b]{2}{*}{ Intervention... } & \multicolumn{2}{|c|}{ HIGH-FAT } & \multicolumn{2}{|c|}{ LOW-GI } & \multicolumn{2}{|c|}{ SUCROSE } & \multicolumn{2}{|c|}{ HIGH-GI } \\
\hline & Median & $95 \% \mathrm{Cl}$ & Median & $95 \% \mathrm{Cl}$ & Median & $95 \% \mathrm{Cl}$ & Median & $95 \% \mathrm{Cl}$ \\
\hline Day 1 & 59 & 39,186 & 116 & 90,281 & 155 & 69,410 & 267 & 160,418 \\
\hline Day 24 & 36 & $2 \cdot 4,108$ & 75 & 37,243 & 132 & 82,188 & 276 & 187,430 \\
\hline Percentage change & -61 & $-119,136$ & -43 & $-114,31$ & -20 & $-112,135$ & $+31^{\star}$ & $-14,142$ \\
\hline
\end{tabular}

HIGH-FAT, $50 \%$ fat (>34\% monounsaturated fat) diet; LOW-GI, high-carbohydrate low glycaemic index diet; HIGH-GI, high-carbohydrate high glycaemic index diet; SUCROSE, high sucrose diet with high carbohydrate increase of $90 \mathrm{~g}$ sucrose/d.

* Median value was significantly different from those of the other three diets (non-parametric ANOVA) $(P<0.001)$.

†For details of diets and procedures, see Table 1 and p. 208. 


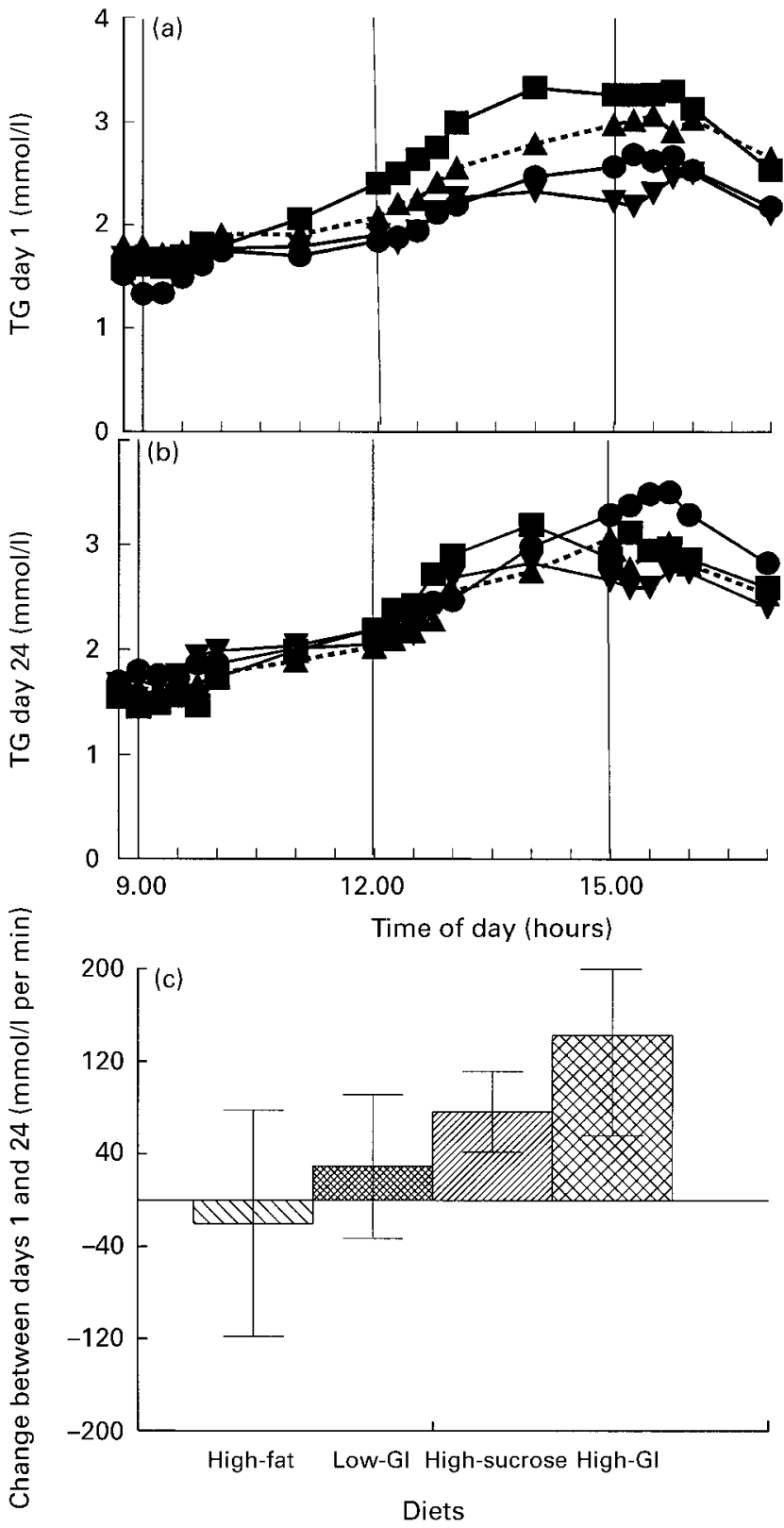

Fig. 3. Circulating plasma triacylglycerol (TG) response to three meals on day 1 (a) and day 24 (b) in men on a high-fat diet ( $\square$ ), a high-glycaemic index (Gl) diet $(\mathbf{\nabla})$, a high-sucrose diet $(\boldsymbol{\bullet})$ or a lowGl diet (ム). (c), Difference between day 1 and day 24. Mean values are shown and vertical bars represent standard errors of the mean. For details of diets and procedures, see Table 1 and p. 208.

performing simple substitutions between commonly used food choices. The present study shows that meaningful dietary changes are achievable outside the laboratory setting.

A strength of the study design is that it compared four diets at once, something that is not typical in nutrition research. All seventeen subjects received all four diets in a randomised 4-way crossover design. Some key foods were provided in addition to two methods of assessing dietary compliance: the return of unused key foods and a $7 \mathrm{~d}$ diary at the end of each intervention. Day profiles over $8 \mathrm{~h}$ with frequent measurements of glucose, insulin, TG
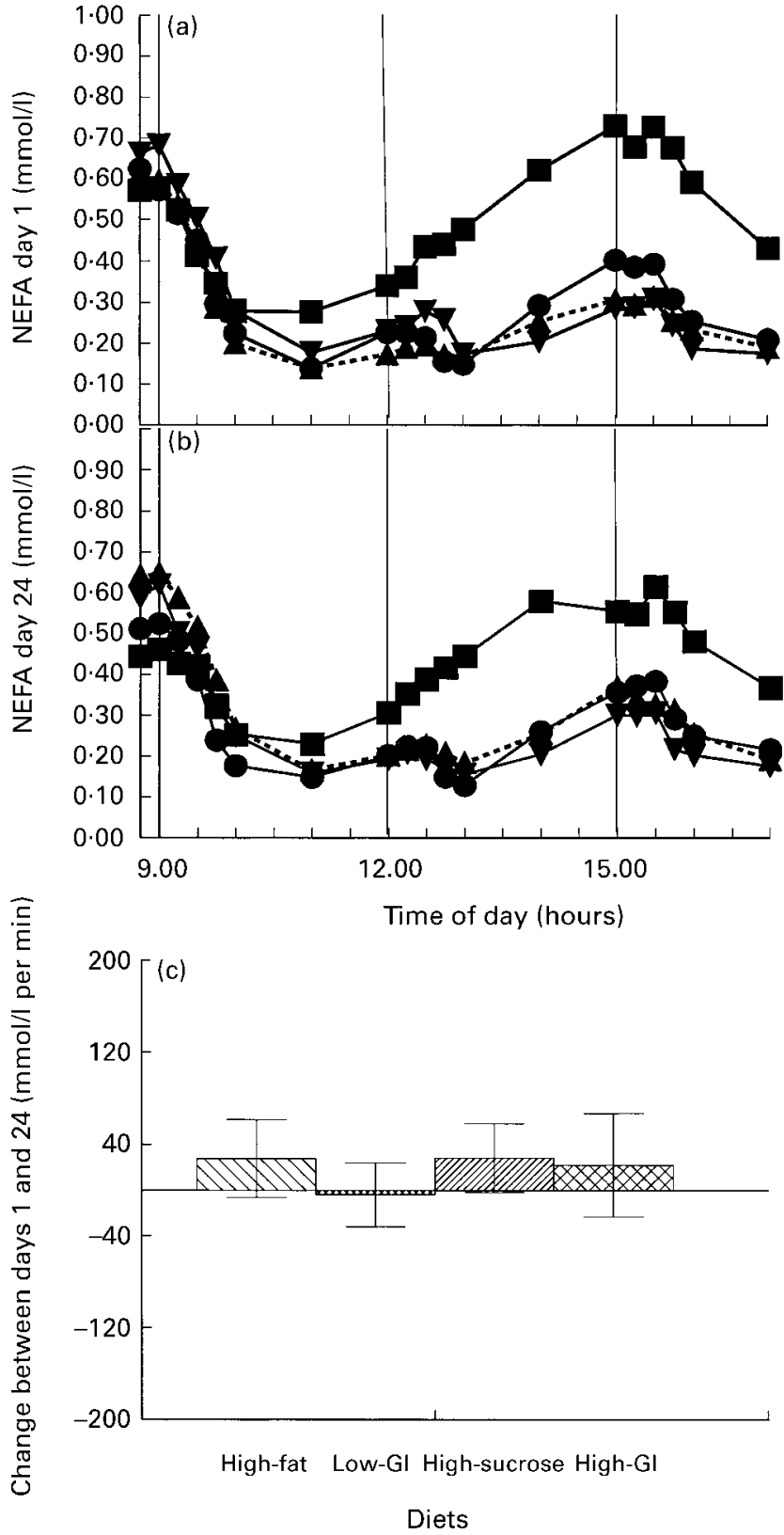

Fig. 4. Circulating plasma non-esterified fatty acid (NEFA) response to three meals on day 1 (a) and day 24 (b) in men on a high-fat diet $(\mathbf{\square})$, a high-glycaemic index (GI) diet ( $\mathbf{\nabla})$, a high-sucrose diet $(\bullet)$ or a low-GI diet ( $\mathbf{\Lambda})$. (c), Difference between day 1 and day 24. Mean values are shown and vertical bars represent standard errors of the mean. For details of diets and procedures, see Table 1 and p. 208.

and NEFA were made at the beginning and the end of each diet period.

In the present study the four intervention diets had no effects on fasting metabolic parameters, having all their effects on postprandial parameters. The significant differences in postprandial insulin concentrations suggest that despite only seventeen volunteers completing the study it was not underpowered.

The high-fat (MUFA) low-carbohydrate diet (30\% carbohydrate) produced lower postprandial glucose and insulin concentrations both at day 1 and after $24 \mathrm{~d}$ than 


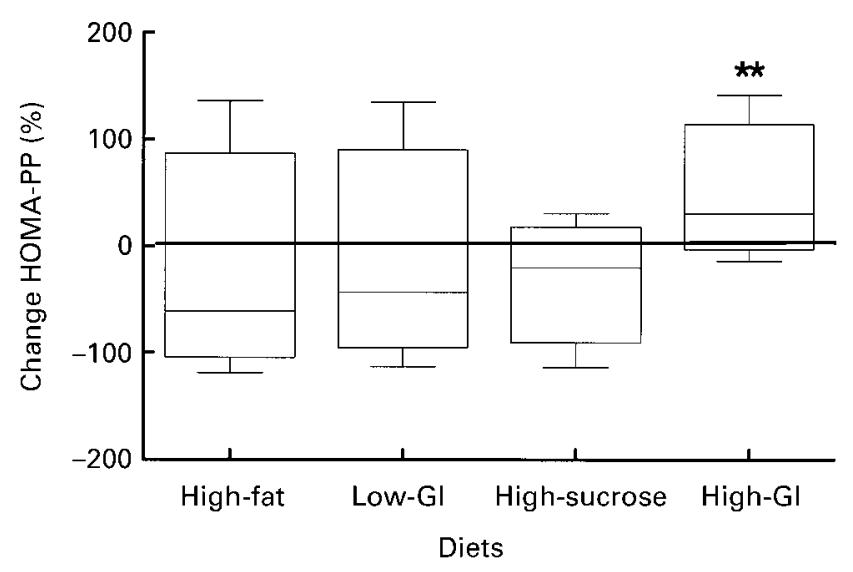

Fig. 5. Percentage change in postprandial homeostatic assessment model (HOMA-PP) (calculated HOMA-PP $\left(\times 10^{3}\right)=$ incremental area under the curve (IAUC) glucose $(\mathrm{mmol} / \mathrm{I} \times \mathrm{min}) \times \mathrm{IAUC}$ insulin/22.5 $(\mathrm{mU} / \mathrm{I} \times \mathrm{min})$ ) response to a high-fat, low-glycaemic index (GI), high-sucrose or high-Gl diet in healthy middle-aged men ( $n$ 17). Mean values are shown and vertical bars represent standard errors of the mean. ${ }^{* *}$ Mean value was significantly different to those for the other three diets $(P<0.01)$. For details of diets and procedures, see Table 1 and p. 208.

the diets that contained less fat but higher carbohydrate loads. It is possible that some if not all of these metabolic benefits that are derived from this diet are attributable to the low carbohydrate load of the high-fat diet. These potential benefits need to be considered alongside the potential atherogenic and thrombogenic consequences of a high-fat low-carbohydrate diet, as postprandial TG values were significantly higher on the high-fat diet and NEFA concentrations failed to be suppressed to the extent seen with the high-carbohydrate interventions. Raised postprandial TG and NEFA has been proposed as an independent risk factor for CHD risk (Hokanson \& Austin, 1996; Williams, 1997).

The study cohort chosen were sedentary middle-aged men with one or more traditional cardiac risk factors. Although healthy at the time of the study their calculated risk of developing CHD over the next 10 years was $11 \%$ (Wood et al. 1998). This population was specifically chosen as it was thought that such a group would benefit the most from targeted lifestyle interventions to reduce CHD.

The dietary changes made in the present study were all realistically achievable in this free-living population as only food choices involving commonly available foods in the UK were promoted. Despite intensive dietetic counselling at the start and throughout each intervention aimed at subjects to maintain a constant energy intake there was a spontaneous energy increase on the HIGH-FAT diet and a tendency for energy intake to reduce on the LOW-GI diet. This resulted in a small but significant weight loss on the LOW-GI diet compared with the high-SUCROSE diet although both the HIGH-FAT and the HIGH-GI subjects moved into positive energy balance. There are many reports that low-GI foods are more filling, making them more useful for individuals who want to lose weight. These reports were reviewed by Ludwig (2000), who concluded that 'although controlled clinical trials of a low glycaemic diet in the treatment of obesity are needed ... a growing body of theoretical and experimental work suggests low GI diets may improve access to stored metabolic fuels, decrease hunger and promote weight loss'.

Prospectively, additional short-term benefits of low-glycaemic diets have included improvements in coagulation (Frost et al. 1994) with reduced concentrations of plasminogen activator inhibitor compound-1 (Jarvi et al. 1999), and fall in blood pressure (Sciarrone et al. 1993).

The GI of the high-SUCROSE intervention fell between that of the LOW-GI and the HIGH-GI diets. On day 1 the TG or NEFA response to the SUCROSE diet was similar to that of the LOW-GI and HIGH-GI intake. This confirms the findings of a previous study of ours (Brynes et al. 2002). By day 24 although there was no change in the relative increase compared with the LOW-GI or HIGH-GI response there was a significant increase in total postprandial TG concentrations and the $6 \mathrm{~h}$ postprandial concentration compared with day 1 . These postprandial TG concentrations were, however, $10 \%$ lower than those seen with the HIGH-FAT intervention and were not significantly different from the other carbohydrate diets. The high sucrose intake in the present study is well above that of the typical UK intake. Despite this, fasting TG remained unchanged and the increased postprandial IAUC after $24 \mathrm{~d}$ ad libitum consumption remained lower than that observed with the HIGH-FAT diet.

The metabolic differences between the LOW-GI and HIGH-GI conditions were apparent from day 1 with a significant difference in insulin that remained on day 24. The LOW-GI diet consumed over $24 \mathrm{~d}$ did not affect postprandial insulin, glucose, TG or NEFA.

The individual metabolic profiles over the $8 \mathrm{~h}$ for each dietary intervention were studied for each meal. Within each intervention each meal had a similar energy and macronutrients content although the foods that these came from were different (Table 1). For each dietary intervention the peak insulin and glucose responses were significantly higher for breakfast than for lunch or afternoon tea, suggesting insulin sensitivity, confirmed using the postprandial HOMA-PP data, is lowest in the morning and increases irrespective of the type of diet throughout the day. The diurnal variation in hormones especially cortisol and growth hormones, which are highest during sleep, may go some way to explaining this.

The present study examines the effects of modifying the fat, GI and sucrose content of the diet on metabolic cardiovascular risk factors. The short- and adaptive medium-term effects of these different diets on postprandial glucose, insulin, TG and NEFA were studied along with the cumulative meal effect of each diet. Despite being advised to maintain identical energy intake there was weight loss on the LOW-GI diet.

In conclusion HIGH-FAT (MUFA) had a beneficial effect on postprandial glucose and insulin over time but it was associated with higher postprandial concentrations of TG and NEFA. Conversely the HIGH-GI diet appeared to increase postprandial insulin resistance over the study period. We believe this present study provides powerful clinical evidence to support the growing epidemiological evidence that the current epidemic of cardiovascular 
disease in the western world is linked with dietary factors and these are amenable to dietary intervention.

\section{Acknowledgements}

We are grateful to the Sugar Bureau for their financial support. We thank Miss Michelle Sapowski of Surrey University for the measurement of plasma TG and NEFA. Caroline Doré from the Medical Statistics Department, Imperial College School of Medicine gave statistical advice. C.M.B.E. is a Diabetes UK RD Lawrence research fellow.

\section{References}

Albano JD, Ekins RP, Maritz G \& Turner RC (1972) A sensitive, precise radioimmunoassay of serum insulin relying on charcoal separation of bound and free hormone moieties. Acta Endocrinol (Copenhagen) 70, 487-509.

Brynes AE, Edwards CMB, Ghatei MA, Bloom SR \& Frost GS (2002) Men at increased risk of coronary heart disease are not different to age and weight matched healthy controls in their postprandial triacylglyceride, non-esterified fatty acid or incretin response to sucrose. Metabolism 51, 195-200.

Coulston AM, Hollenbeck CB, Swislocki AL, Chen YD \& Reaven GM (1987) Deleterious metabolic effects of highcarbohydrate, sucrose-containing diets in patients with noninsulin-dependent diabetes mellitus. American Journal of Medicine 82, 213-220.

Coulston AM, Hollenbeck CB, Swislocki AL \& Reaven GM (1989) Persistence of hypertriglyceridemic effect of low-fat high-carbohydrate diets in NIDDM patients. Diabetes Care 12, 94-101.

Department of Health (1994) Committee on Medical Aspects of Food Policy. Nutrition Aspects of Cardiovascular Disease. London: HMSO.

Despres J-P, Lamarche P, Mauriege P, Cantin B, Dagenais GR, Sital M \& Lupien P-J (1996) Hyperinsulinemia as an independent risk factor for ischemic heart disease. New England Journal of Medicine 334, 952-957.

FAO/WHO (1998) Carbohydrates in human health. Paper 66. Report of a joint FAO/WHO report Rome 14-18 April 1997. FAO Food and Nutrition.

Fontbonne A, Charles MA, Thibult N, Richard JL, Claude JR, Warnet JM, Rosselin GE \& Eschwege E (1991) Hyperinsulinaemia as a predictor of coronary heart disease mortality in a healthy population: the Paris Prospective Study, 15-year follow-up. Diabetologia 34, 356-361.

Foster-Powell K \& Miller JB (1995) International tables of glycaemic index. American Journal of Clinical Nutrition 62, 871S-893S

Frayn KN \& Kingman SM (1995) Dietary sugar and lipid metabolism in humans. American Journal of Clinical Nutrition $\mathbf{6 2}$, 250s-263s.

Frost G, Leeds AA, Dore CJ, Madeiros S, Brading S \& Dornhorst A (1999) Glycaemic index as a determinant of serum HDLcholesterol concentration. Lancet 27, 1045-1048.

Frost G, Wilding J \& Beecham J (1994) Dietary advice based on the glycaemic index improves dietary profile and metabolic control in type 2 diabetic patients. Diabetic Medicine 11, $397-401$.

Garg A (1994) High-monounsaturated fat diet for diabetic patients. Is it time to change the current dietary recommendations? Diabetes Care 17, 242-246.
Garg A, Bantle JP, Henry RR, Coulston AM, Griver KA, Raatz SK, Brinkley L, Chen YD, Grundy SM \& Huet BA, et al. (1994) Effects of varying carbohydrate content of diet in patients with non-insulin-dependent diabetes mellitus. Journal of the American Medical Association 271, 1421-1428.

Gillum RF (1994) Trends in acute myocardial infarction and coronary heart disease death in the United States. Journal of the American College of Cardiology 23, 1273-1277.

Hokanson JE \& Austin MA (1996) Plasma triglyceride level is a risk factor for cardiovascular disease independent of HDL cholesterol level: a meta-analysis of population based prospective studies. Journal of Cardiovascular Risk 3, 213-219.

$\mathrm{Hu}$ F, Stampfer MJ, Manson JE, Grodstein F, Colditz GA, Speizer FE \& Willett WC (2000) Trends in the incidence of coronary heart disease and changes in diet and lifestyle in women. New England Journal of Medicine 383, 530-537.

Jarvi AE, Karstrom BE, Granfeldt Y, Bjorck I, Asp NG \& Vessby B (1999) Improved glycemic control and lipid profile and normalized fibrinolytic activity on a low-glycaemic index diet in type 2 diabetes. Diabetes Care 22, 10-18.

Jenkins DJ, Wolever TM, Taylor RH, Barker H, Fielden H, Baldwin JM, Bowling AC, Newman HC, Jenkins AL \& Goff DV (1981) Glycemic index of foods: a physiological basis for carbohydrate exchange. American Journal of Clinical Nutrition 34, 362-366.

Levy J, Matthews DR \& Hermans MP (1998) Correct homeostasis model assessment (HOMA) evaluation uses the computer program. Diabetes Care 21, 2191-2192.

Liu S, Willett WC, Stampfer MJ, Hu FB, Franz M, Sampson L, Hennekens CH \& Manson JE (2000) A prospective study of dietary glycaemic load, carbohydrate intake, and risk of coronary heart disease in US women. American Journal of Clinical Nutrition 71, 1455-1461.

Ludwig DS (2000) Dietary glycaemic index and obesity. Journal of Nutrition 130, 280S-283S

Ludwig DS (2002) The glycaemic index: Physiological mechanisms relating to diabetes and cardiovascular disease. Journal of the American Medical Association 287, 2414-2423.

McKeigue PM, Ferrie JE, Pierpoint T \& Marmot MG (1993) Association of early-onset coronary heart disease in South Asian men with glucose intolerance and hyperinsulinemia. Circulation 87, 152-161.

Mensink RP \& Katan MB (1990) Effect of dietary trans fatty acids on high-density and low-density lipoprotein cholesterol levels in healthy subjects. New England Journal of Medicine 323, 439-445.

Murray CJL \& Lopez AD (1997) Mortality by cause for eight regions of the world: Global Burden of Disease. Lancet 349, $1269-1276$.

Parks EJ \& Hellerstein MK (2001) Carbohydrate-induced hypertriacylglycerolemia: historical perspective and review of biological mechanisms. American Journal of Clinical Nutrition 71, 412-433.

Raben A, Holst J, Madsen J \& Astrup A (2001) Diurnal metabolic profiles after $14 \mathrm{~d}$ of an ad libitum high starch, high sucrose or high fat diet in normal-weight never obese and postobese women. American Journal of Clinical Nutrition 73, 177-189.

Reaven GM (1995) The fourth musketeer - from Alexandre Dumas to Claude Bernard. Diabetologia 38, 3-13.

Reaven GM (1997) Do high carbohydrate diets prevent the development or attenuate the manifestations (or both) of syndrome $\mathrm{X}$ ? A viewpoint strongly against. Current Opinion in Lipidology 8, 23-27.

Sciarrone SE, Strahan MT, Beilin LJ, Burke V, Rogers P \& Rouse IR (1993) Ambulatory blood pressure and heart rate responses to vegetarian meals. Journal of Hypertension 11, 277-285.

Turner RC, Holman RR, Matthews DR, Hockaday TD \& Peto J 
(1979) Insulin deficiency and insulin resistance interaction in diabetes: estimation of their relative contribution by feedback analysis from basal plasma insulin and glucose concentrations. Metabolism 28, 1086-1096.

Williams CM (1997) Postprandial lipid metabolism: effects of dietary fatty acids. Proceedings of the Nutrition Society 56, 679-692.

Wood D, Durrington P, Poulter N, McInnes G, Rees A \& Wray R (1998) Joint British recommendations on prevention of coronary heart disease in clinical practice. Heart 80, s1-s26. 